\title{
Study of Radiation Interaction Mechanisms of Different Nuclear Detectors
}

\author{
M. N. Islam ${ }^{1}$, H. Akhter ${ }^{2}$, M. Begum ${ }^{3}$, M. S. Alam ${ }^{4}$ and M. Kamal ${ }^{5}$ \\ ${ }^{1,2,3}$ Electronics Division, Atomic Energy Centre, Bangladesh Atomic Energy Commission, P.O. Box No. 164, Dhaka, Bangladesh. \\ ${ }^{4}$ Institute of Electronics, Atomic Energy Research Establishment, Bangladesh Atomic Energy, Commission, G.P.O Box 3787, Savar, \\ Dhaka, Bangladesh. \\ ${ }^{5}$ Phy sical Science Division, Bangladesh Atomic Energy Commission, P.O. Box No. 158, Dhaka, Bangladesh. \\ E-mail: nislam_baec@yahoo.com
}

\begin{abstract}
In this paper, an attempt has been made to describe the radiation interaction mechanisms ofnuclear detectors. There are lots of radioactive detectors available in the field of radiation detection and measurements instruments/systems such as Geguier Muller (GM) Tube, Scintillation Counter, High Purity Germanium (HPGe) and so on. Each of these detectors have different and distinct radiation interaction mechanisms and detecting principle for processing each type of radiation measurement (qualititative and quantitative).The interaction mechanisms of these detectors are governed by generation of ions (positive and negative) in case of GM tube; the photo-electric effect, Compton scattering and pair production for Scintillation detectorand HPGe along with diode principle. The special feature of this diode is a constant current generator depending on the energy of the photon deposition in the detector. The characteristics of these interaction mechanisms have been presented along with intensity of measurements, efficiency and detector resolution (FWHM).

Keywords-Radiation Interactions, Radiation Measurements, Nuclear Detectors, Photo-electric Effect Compton Scattering and Pair Production.
\end{abstract}

\section{INTRODUCTION}

Nuclear detectors are devices that convert the energy of a photon or incident particle into an electric pulse [1].These detectors fall into two categories: gross counters and energy sensitive. Gross counters count each event (gamma or neutron) emission the same regardless of energy. Energy sensitive detectors, used in radio-isotope identification devices (RIIDs), analyze a radioactive is otope's distinct gamma energy emissions and attempt to identify the source of the radiation [2]. Gamma rays and $\mathrm{x}$-rays ionize the gas indirectly by interacting with the metal wall of the GM tube via the photoelectric effect, Compton scattering or pair production in such a way that an electron is "knocked" off the inner wall of the detector. The electric field created by the potential difference between the anode and cathode causes the negative member of each ion pair to move to the anode while the positively charged gas atom or molecule is drawn to the cathode. If the electric field in the chamber is of sufficient strength approximately $10^{6} \mathrm{~V} / \mathrm{m}$ these electrons gain enough kinetic energy to ionize the gas and create secondary ion pairs. The result is that each electron from a primary ion pair produces a cascade or avalanche of ion pairs [3]. When ionizing radiation enters a scintillator, it produces a fluorescent flash with short decay time. This is known as scintillation. In the case of gamma rays, this scintillation occurs as a result of excitations of bound electrons by means of free electrons in the scintillator. These free electrons are generated by following three mutual interactions photoelectric effect, Compton scattering or pair production. The probability of occurrence of such interactions depends on type of scintillator and the energy of the Gamma rays [4]. The purpose of an HPGe detector is to convert gamma rays into electrical impulses which can be used, with suitable signal processing, to determine their energy and intensity. All HPGe radiation detectors are either coaxial HPGe, well-type HPGe or broad energy HPGe (BEGe) just large, reverse-biased diodes. The germanium material can be either "n-type" or "p-type". The type depends on the concentration of donor or acceptor atoms in the crystal[5].

\section{METHODOLOGY}

2.1 Gamma Interaction Mechanism

Four major interaction mechanisms play an important role in the measurement of photons. These mechanisms are: photoelectric effect, coherent scattering, incoherent scattering and pair production. The photon energy of major interest for environmental spectrometry studies ranges between a few $\mathrm{keV}$ and $1500 \mathrm{keV}$. The term "low energy" will be used here for the energy range 1 to 100 $\mathrm{keV}$, "medium energy" for energies between 100 and 600 
$\mathrm{keV}$ and "high energy" for energies between 600 and $1500 \mathrm{keV}$.

2.1.1: Photoelectric Effect

In the photoelectric effect, there is a collision between a photon and an atom resulting in the ejection of a bound electron. The photon disappears completely, i.e. all its energy, Ep, is transferred to the electron. The amount of energy, Ee, which is transferred to the electron can be calculated if the binding energy, $\mathrm{Eb}$, of the ejected electron is known:

$$
\mathrm{Ee}=\mathrm{Ep}-\mathrm{Eb}
$$

\subsection{2: Coherent Scattering}

In the coherent scattering process no energy is transferred to the atom. The electromagnetic field of the photon sets atomic electrons into vibration. The electrons then re-emit radiation of the same magnitude as the interacting photon and mainly in the forward direction. The cross-section for coherent scattering decreases rapidly with increasing photon energy. This process can be neglected for photon energies above $100 \mathrm{keV}$. The differential cross section per atom for this process as a function of scattering angle $\theta$ is written as follows:

$$
\frac{\partial \sigma_{\operatorname{coh}}}{\partial \theta}=\frac{z r_{0}{ }^{2}}{2}\left(1+\cos ^{2}(\theta)\right)[F(x, Z)]^{2} \cdot 2 \pi \cdot \sin (\theta)
$$

with the parameter $\mathrm{x}$ defined as:

$$
x=\frac{\sin (\theta / 2)}{\lambda}
$$

Where $\mathrm{r}_{0}$ is the classical electron radius, $\mathrm{F}(\mathrm{x}, \mathrm{Z})$ is the atomic form factor and $\lambda$ is the photon wavelength.

\subsection{3: Incoherent or Compton scattering}

In the incoherent or Compton scattering process, only a portion of the photon energy is transferred to an electron. The remaining energy appears as a secondary photon. The direction (scattering angle theta) and energy of the secondary photon, Ep', are related by the following equation:

$$
E_{p}^{\prime}=\frac{E_{p}}{1+\alpha(1-\cos (\theta))}
$$

With $\alpha$ defined as

$$
\alpha=\frac{E_{p}}{m_{\mathrm{o}} c^{2}}
$$

Where $m_{0}$ is the rest mass for an electron and $c$ the speed of light in vacuum.

2.1.4: Pair Production
The pair production mechanism only occurs for photon energies above $1022 \mathrm{keV}$. Here photons are converted to electron-positron pairs under the effect of the field of a nucleus. Since one electron and one positron are formed, the photons must have energies equivalent to at least two electronic masses $(2 \times 511 \mathrm{keV})$ and the excess photon energy is shared between the created electron and positron pair. The annihilation of the positrons produces two photons in opposite directions, each with $511 \mathrm{keV}$. The total cross-section for this process increases with energy above the threshold energy.

$$
\sigma_{\text {Total }}=\sigma_{\text {Photollectric }}+\sigma_{\text {Pair Production }}+\sigma_{\text {Coherent Sa, }}+\sigma_{\text {Incoherent Sa, }} \text { (6) }
$$

The total probability of interaction per unit path length for a photon is proportional to the sum of the total individual cross-sections [6].

\section{2: Detector Bias Power Supply}

Generally it is a high voltage power supply with enough current capacity to feed the detector without any loose in regulation. The ripple should be less than $100 \mathrm{mV}$. For stable operation of the detector in any measurement system, it needs bias voltages ranges from $400-1000 \mathrm{~V}$ for GM Tube; $1000-3000 \mathrm{~V}$ for Scintillation and 3000-5000V for HPGe.

\section{3: Count Rate Consideration}

Generally, a $\gamma$-radiation from a ${ }^{60} \mathrm{Co}$ source is specified to characterize the almost all types of detectors. A source with an activity of around $37000 \mathrm{~Bq}(1 \mu \mathrm{Ci})$ could be used. The choice of detector in any count rate application depends not only on the systemelectronics but also on the count rates. The count rate can be classified as below:

$$
\begin{aligned}
& \text { * Low - Below } 100 \mathrm{cps} \\
& \text { * High - Above 75,000 cps input rate } \\
& \text { * Very High - Above 100,000 cps [5] }
\end{aligned}
$$

The GM tube is suitable for the first count rate; scintillation for the second and HPGe for low to very high.

\section{4: Detector Sensitivity \& efficiency}

\subsection{1: Sensitivity}

The sensitivity, S, to $\gamma$ - $\square$ radiation is defined as the ratio of the number of counts per second, $\mathrm{N}$, obtained and the exposure rate, $X$.

$$
S=\frac{N}{X}
$$


Generally, it is specified for a Cobalt-60 source produces an exposure rate $X$ in $\mathrm{R} / \mathrm{hr}$. The sensitivity can vary widely, between $0.2 \mathrm{cps} / \mathrm{mR} / \mathrm{hr}$ and $240 \mathrm{cps} / \mathrm{mR} / \mathrm{hr}$, depending on the detector model [1].

2.4.2: Quantum efficiency and Collection efficiency

The photomultiplier tube output current Ip of NaI (T1) Scintillator is given by

$$
I_{p}=\frac{N \times \eta \times \alpha \times \mu \times e}{\tau_{s}}
$$

Where

$\mathrm{N}=$ Amount of light flash per event produced from scintillator

$\eta=$ Quantum efficiency of photocathode (assumed to be $25 \%)$

$\alpha=$ Collection efficiency of photomultiplier tube (assumed to be $90 \%$ )

$\mu=$ Gain of photomultiplier tube

$\mathrm{e}=$ Electron charge

$\tau \mathrm{s}=$ Decay time of NaI $(\mathrm{TI})$

From Eq. 2.4.2 Quantum efficiency of photocathode and Collection efficiency of photomultiplier tube can be derived as follows[4]:

$$
\eta=\frac{I_{p} \times \tau_{s}}{N \times \alpha \times \mu \times e}
$$

and

$$
\alpha=\frac{I_{p} \times \tau_{s}}{N \times \eta \times \mu \times e}
$$

\subsection{3:Absolute efficiency}

The absolute detector efficiency at that energy is calculated by dividing the net count rate in the full-energy peak by the decay corrected gamma-ray-emission rate of the standard source. Efficiency curves were constructed from these full-energy-peak efficiencies.

$\varepsilon_{a b s}=\frac{\text { Total number of counts recorded under the photo peak }}{\text { Total number of photons emitted by the standard sources }}$

$\varepsilon_{a b s}=\frac{\text { cps experimental }}{\text { cps theoritical }}$

$\varepsilon_{a b s}=\frac{\left(\frac{\mathrm{C}}{\mathrm{S}}\right)_{\mathrm{std}}-\left(\frac{\mathrm{C}}{\mathrm{S}}\right)_{\mathrm{sample}}-\left(\frac{\mathrm{C}}{\mathrm{S}}\right)_{\mathrm{BG}}}{\left(\frac{\mathrm{C}}{\mathrm{S}}\right)_{\text {theo }} \operatorname{Exp}\left(-\ln (2) \cdot \frac{\mathrm{t}}{\mathrm{t}_{1}}\right)}$
$\left(\frac{\mathrm{C}}{\mathrm{S}}\right)$ std Count of soil sample with standard solution, $\left(\frac{\mathrm{C}}{\mathrm{S}}\right)$ sample Count of soil sample without standard solution, $\left(\frac{\mathrm{C}}{\mathrm{S}}\right)$ BG Count of background, $\left(\frac{C}{S}\right)$ theo, Counting of gamma ray of used standard solution, $\mathrm{t}$ is the time of decay, $\mathrm{t}_{1 / 2}$ is half-life of the radionuclide [7].

2.5: Detector Resolution

2.5.1:Scintillation Counting

There are two measurement methods available in Scintillation Counting. One is spectrum method and the other is counting method. In the spectrum method, pulse height discrimination is important to determine photopeaks produced by various types of radiation. This is evaluated as Energy Resolution or Pulse Height Resolution(PHR).

The energy resolution is defined by the following equation as shown fig.1.It is generally expressed as a percentage.

$$
R=\frac{\Delta P}{P}
$$

$\mathrm{R}=$ Energy Resolution

$\mathrm{P}=$ Peak Value

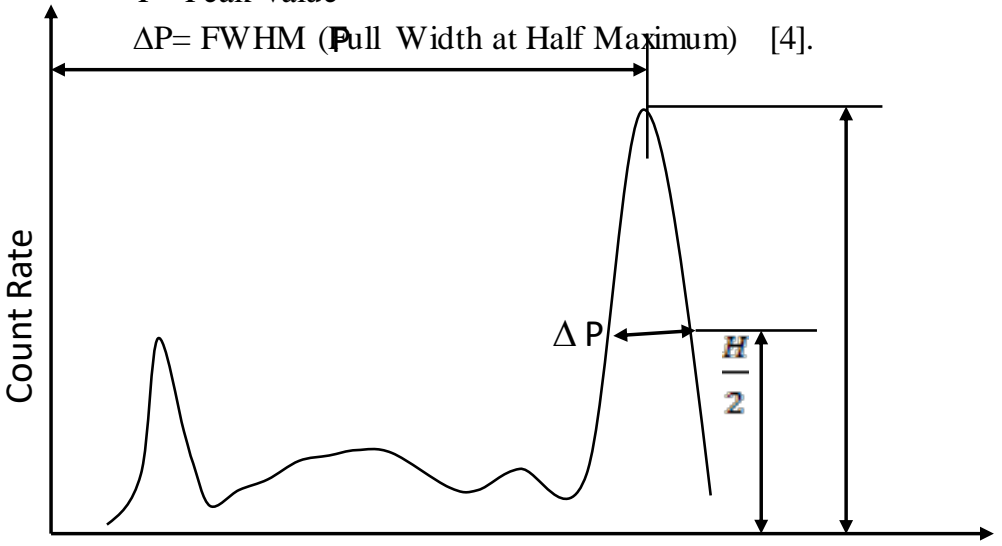

Pulse Height (Energy)

Fig.1: Definition of Energy Resolution.

2.5.2: High Purity Germanium (HPGe)

Energy resolution is the dominant characteristic of a germanium detector. Gamma-ray spectrometry using high purity germanium detector is enhanced by the excellent energy resolution which can help to separate and resolve various close energy gamma-ray peaks in a complex energy spectrum. The full width at half maximum of the full energy peak known as FWHM and sometimes referred as a measure of energy resolution. The units of FWHM are expressed in $\mathrm{KeV}$ for $\mathrm{Ge}$ detector and are defined at specific, characteristic full energy peaks associated with standard sources such as $662 \mathrm{KeV}$ for a 
${ }^{137} \mathrm{Cs}$ source or $1332 \mathrm{KeV}$ for a ${ }^{60} \mathrm{Co}$ source. The energy resolution of the germanium detector can affected by the number of electron-hole pairs created in the detector, incomplete charge collection and electronic noise contributions. The effect of these three factors depends on the properties of the detector and the gamma-ray energy [8].

\section{6: Discussion}

The radiation interaction mechanisms for Geguier Muller (GM) Tube, Scintillation Counter, High Purity Germanium (HPGe) detectors have been presented in this study. Although these three different nuclear detectors having different operating principles and specific applications but the four major interaction mechanisms viz. photoelectric effect, coherent scattering, incoherent scattering and pair production dominate the detection process.

And these interaction mechanisms have been described in sub-section 2.1.The detector bias power supply for these three detectors also vary chronologically from $400 \mathrm{~V}$ to $5000 \mathrm{~V}$ as shown in sub-section 2.2 .In case of count rate consideration for the same detectors, HPGe would be the superior with respect to two others. According to subsection 2.3 , this detector is suitable for both low to very high energy gamma rays, the GM Tube is at the bottom and scintillation counter stands in between position of them. Regarding performance evaluation, the GM tube belongs to sensitivity rather than efficiency

but the terminology 'efficiency' goes well with the rest of two detectors. Quantum efficiency of photocathode and Collection efficiency of photomultiplier tube are important for scintillation counter. The absolute detector efficiency of HPGe is important than intrinsic or relative efficiency.

\section{CONCLUSION}

An elaborate study of radiation interaction mechanisms of different nuclear detectors like GM tube, Scintillation counter and HPGe have been presented in this research. Moreover, range of detector bias voltages and count rate considerations from low to very high energy also have been presented sufficiently. Detector sensitivity and efficiency have been discussed as well. The study would be helpful hints for the design and detecting principle of nuclear detectors.

\section{ACKNOWLEDGEMENTS}

Authors wish to express deep gratitude to Mr. Mahbubul Hoq, Chairman, Mr. Masud Kamal, Member (Physical Science), Dr. Imtiaz Kamal, Member (Planning and
Development), Dr. Md. Sanowar Hossain, Member (BioScience) and Engr. Md. Abdus Salam, Member (Engineering), Bangladesh Atomic Energy Commission, Dhaka for their support and cooperation in the research.

\section{REFERENCES}

[1] Test Procedure for Geiger-Mueller Radiation Detectors, MRNI-501, REV.: D0, December 2008, Available on line 2012.

[2] White Paper, Why High-Purity Germanium (HPGe) Radiation Detection Technology is Superior to Other Detector Technologies for Isotope Identification. http://www.ortec online.com/papers/la_ur_03_4020.pdf. Available online 2013.

[3] M. Nazrul Islam, A.K.M. S. Islam Bhuian, Masud Kamal, Kh. Asaduzzaman1 and Mahbubul Hoq, Design and Development of a 6-Digit Microcontroller Based Nuclear Counting System, International Journal of Scientific Research and Management, Vol. 1, Issue 6, ISSN: 2321 3418, Valley International, September 2013.

[4] Chapter 7, Scintillation Counting, Available on line 2012.

[5] ORTEC, The Best Choice of High Purity Germanium (HPGe) Detector, www.ortec online.com, Available on-line 2013.

[6] F. J. Hernandez "Optimization of environmental gamma spectrometry using Monte Carlo methods" Ph.D. thesis, Uppsala University, Sweden. 2002.

[7] S. Harb, K. Salahel Din and A. abbady, Study of Efficiency Calibrations of HPGe Detectors for Radioactivity Measurements of Environmental Samples, Proceedings of the $3^{\text {rd }}$ Environmental Physics Conference, Aswan, Egy pt- 207 -19-23 Feb. 2008.

[8] Nurul Absar "Study of the Radioactivity in Soil and Tea Leaf and Transfer Factor of Radionuclides", M.Phil. thesis, Chittagong University, Bangladesh, 2012. 